Rev Inv Vet Perú 2018; 29(2): 720-724

http://dx.doi.org/10.15381/rivep.v29i2.14535

\title{
Comunicación
}

\section{Tratamiento quirúrgico temprano de un caso de luxación patelar lateral bilateral en un paciente canino}

\author{
EARLY SURGICAL TREATMENT OF A CASE OF BILATERAL LATERAL PATELLAR LUXATION IN A \\ CANINE PATIENT
}

Ysaac Chipayo G. ${ }^{1,3}$, Carlos Huerta M. ${ }^{1}$, Juan Olazábal L. ${ }^{2}$, Diego Díaz C. ${ }^{1}$

\section{Resumen}

El presente trabajo muestra el caso clínico de un paciente canino hembra de un mes y medio diagnosticado con luxación patelar grave, severa deformidad de miembros posteriores e incapacidad para deambular que fue sometido a corrección quirúrgica con resultados satisfactorios.

Palabras clave: luxación patelar; trocleoplastia; trasposición de cresta tibial; cirugía pediátrica canina

\section{Abstract}

This report shows the clinical case of a female canine patient of 1.5 molth-old diagnosed with severe patellar luxation, severe deformity of the hind limbs and inability to walk, who underwent surgical correction with satisfactory results.

Key words: patellar luxation; trochleoplasty; transposition of tibial tuberosity, canine pediatric surgery

${ }^{1}$ Clínica de Animales Menores, Facultad de Medicina Veterinaria, Universidad Nacional Mayor de San Marcos, Lima, Perú

${ }^{2}$ Laboratorio de Bioquímica, Nutrición y Alimentación Animal, Facultad de Medicina Veterinaria, Universidad Nacional Mayor de San Marcos, Lima, Perú

${ }^{3}$ E-mail: ychipayog@unmsm.edu.pe

Recibido: 13 de octubre de 2017

Aceptado para publicación: 15 de marzo de 2018 


\section{INTRODUCCIÓN}

La luxación patelar es una de las patologías ortopédicas más comunes en ser diagnosticadas en pacientes caninos (Alam et al., 2007). En el medio peruano no existen datos estadísticos de su presentación; sin embargo, datos no publicados de la Clínica Veterinaria de la Universidad Nacional Mayor de San Marcos la sitúan como una de las causas más comunes de cojera de miembros posteriores en la zona de Lima, Perú. Esta enfermedad presenta una alta heredabilidad que predispone a cambios en la forma de la articulación produciendo grados variables de pérdida de contacto de la articulación femoro-patelar (DeCamp y Jhonson, 2006).

El apoyo inadecuado de las fuerzas normales producidas durante el desplazamiento durante el crecimiento del paciente y el desarrollo biomecánico de la articulación facilitan la aparición de anormalidades secundarias y la evolución hacia la enfermedad degenerativa articular crónica (EDAC) en el paciente (Martí, 2013). La edad de diagnóstico de la enfermedad varía según el grado de luxación y los signos clínicos asociados, pero generalmente se presenta entre los tres meses y los 16 años (Hayes et al., 1994; Arthurs y Langley-Hobbs, 2006; Alam et al., 2007); sin embargo, la mayoría de los casos de luxación lateral comienzan a mostrar signos clínicos a los cinco o seis meses de edad (Piermattei et al., 2006).

En el Perú no se realizan planes de búsqueda y control de este tipo de patologías, que pueden ser diagnosticadas en pacientes muy jóvenes, con el fin de evitar la evolución de la enfermedad en etapas posteriores de la vida. Aún más, dadas las complicaciones técnicas de la cirugía en pacientes pequeños y la supuesta complejidad de los protocolos anestésicos, se tiende a postergar la intervención correctiva a etapas posteriores de la vida del paciente (siete meses a un año), cuando complicaciones secundarias de ma- yor gravedad, e incluso EDAC, puedes estar presentes. El objetivo del presente trabajo fue demostrar la factibilidad de la aplicación de las técnicas quirúrgicas de corrección de la luxación patelar en animales jóvenes.

\section{Caso Clínico}

Una paciente canina de aproximadamente mes y medio de edad, raza mestiza, fue recibida en los consultorios externos de la Clínica de Animales Menores de la Facultad de Medicina Veterinaria de la Universidad Nacional Mayor de San Marcos (Lima, Perú), con historia de presentar una severa deformidad de los miembros posteriores que limitaban su desplazamiento y dificultaban su caminar. El propietario mencionó haber consultado previamente con dos especialistas que le habían recomendado iniciar posibles tratamientos con una edad más avanzada. El propietario la trae para buscar una tercera opinión.

En la evaluación del desplazamiento, la paciente mostró incapacidad de pararse sobre sus miembros posteriores, así como una aparente desviación valgus marcada, que posicionaba los pies en $90^{\circ}$ al eje longitudinal del cuerpo.

A la evaluación directa de las rodillas del paciente se evidenció una angulación inadecuada de la rodilla hacia lateral y un cuadro de luxación patelar lateral grado 4 (Figura 1). Como parte del protocolo de diagnóstico se recomienda la toma de imágenes radiográficas para definir la existencia de deformidades óseas.

La imagen radiográfica ventrodorsal del paciente con los miembros extendidos muestra estructuras anatómicas aparentemente normales para un paciente de dos meses de edad. Se evidencia aún la presencia de amplias zonas radiolúcidas en las regiones fisiarias y epifisiarias correspondientes a las estructuras cartilaginosas propia de un cachorro en crecimiento (Figura 2). 


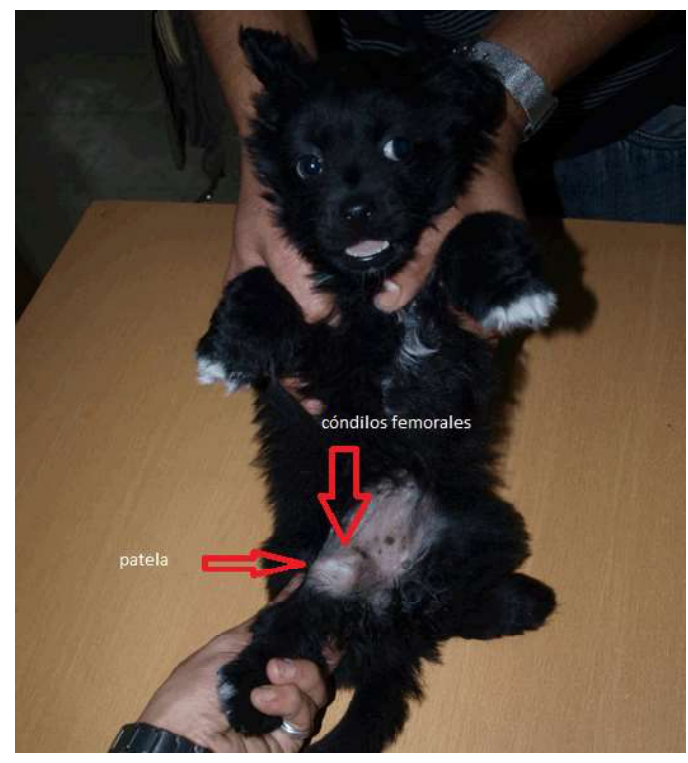

Figura 1. Vista antero posterior de la rodilla del paciente donde se muestra la luxación patelar lateral

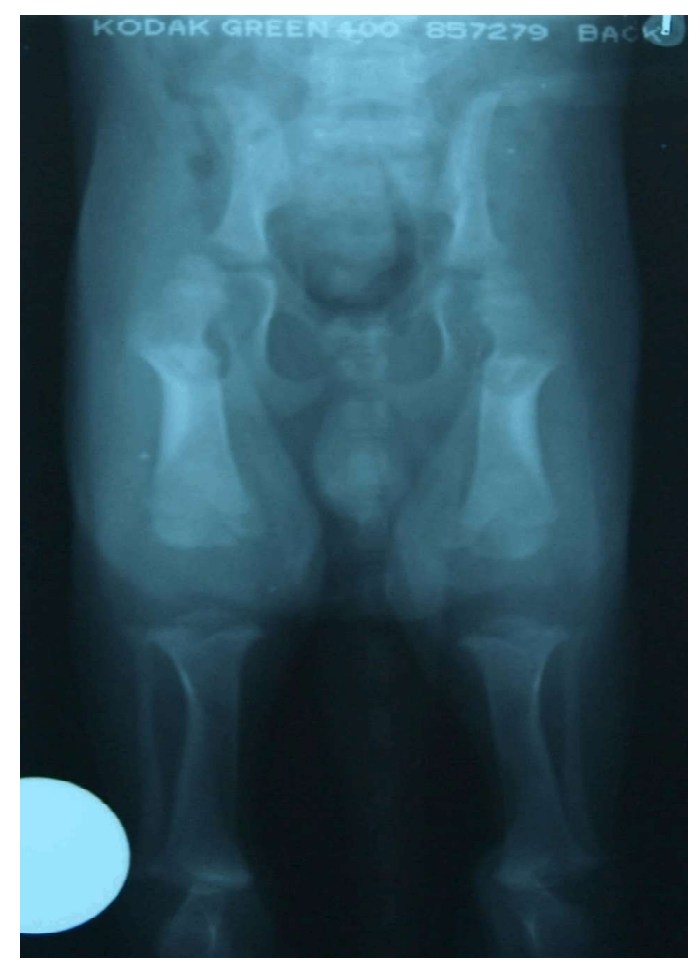

Figura 2. Placa radiográfica ventro-dorsal tipo 1. Nótese el estado inmaduro del esqueleto del paciente con zonas epifisiarias y articulares poco definidas. Además, no se evidencia angulación de estructuras óseas

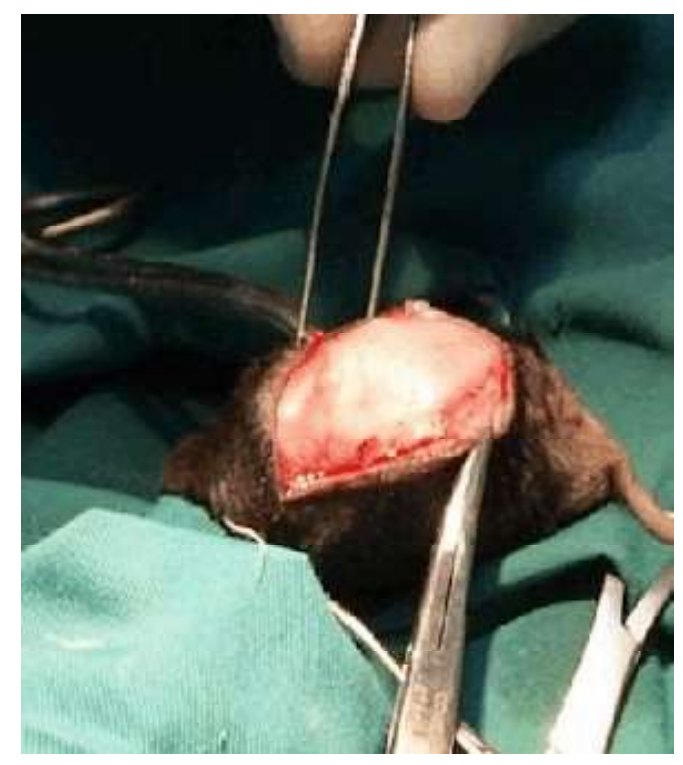

Figura 3. Artrotomía exploratoria donde se observa la ausencia de la concavidad de la corredera troclear

Una vez explicado al propietario sobre los posibles impactos de la enfermedad en el crecimiento y desarrollo del paciente, se decidió proceder a la cirugía exploratoria y correctiva. La paciente fue anestesiada con un protocolo que incluyó diazepam $0.25 \mathrm{mg} / \mathrm{kg}$, fentanilo $5 \mu \mathrm{g} / \mathrm{kg}$ en bolo y $3 \mu \mathrm{g} / \mathrm{kg} / \mathrm{hora}$ en infusión continua y cefalexina $25 \mathrm{mg} / \mathrm{kg}$ como premedicación. La inducción se hizo con ketamina $10 \mathrm{mg} / \mathrm{kg}$ y el mantenimiento del plano anestésico se hizo con isofluorano 1.5-2\%.

El paciente fue sometido a una doble artrotomía de rodilla (Figura 3), observándose la ausencia del surco de la corredera troclear y una angulación moderada de la estructura de la cresta tibial, por lo que se realizaron las técnicas de trocleoplastia en cuña (Figura 4) y la transposición de la cresta tibial. Finalmente se hizo la liberación del retináculo extensor lateral y la imbricación del retináculo medial. Inmediatamente después de la cirugía se colocó un vendaje de 

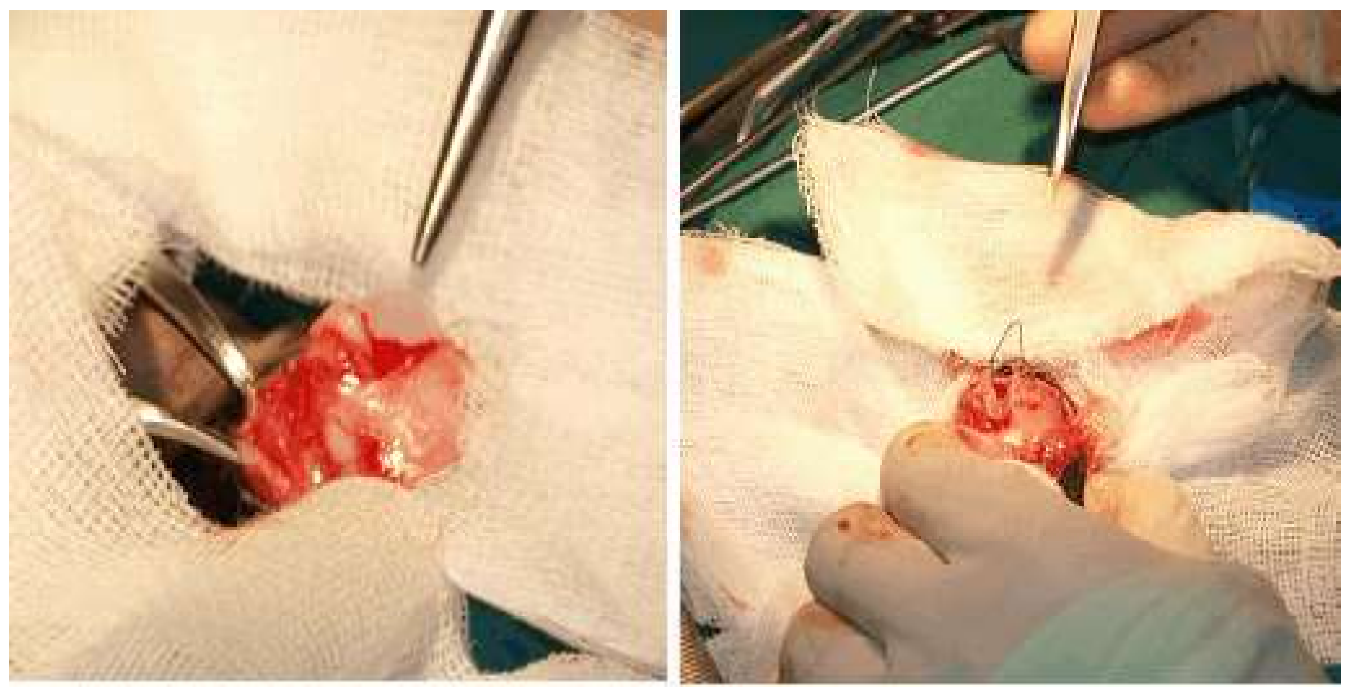

Figura 4. Marcaje de las zonas donde se debe formar los labios de la corredera troclear durante la técnica de trocleoplastia en cuña (izquierda). Aspecto final de la corredera troclear mostrando la profundización lograda durante el procedimiento (derecha)

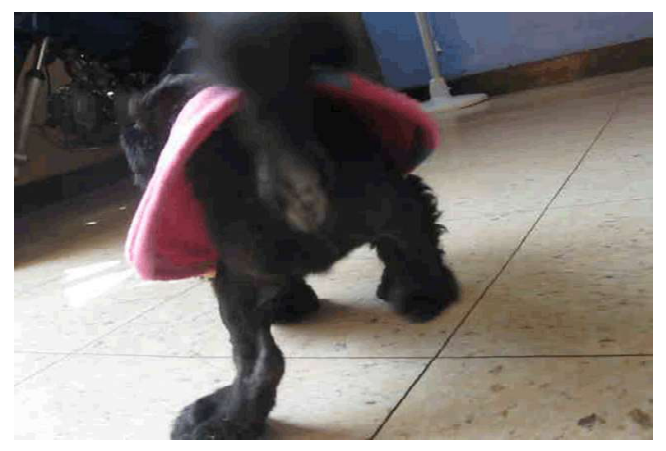

Figura 5. Imagen del paciente el día 15 posquirúrgico

Robert Jhones, en ambos miembros intervenidos, indicando al propietario que los retire cuatro días después. En la Figura 5 se presenta la evaluación del paciente el día 15 posquirúrgico. Se observa la notoria mejoría de la posición de ambos pies y una correcta alineación de los miembros posteriores. Nótese el apoyo de peso sobre los miembros posteriores.
A pesar que la tendencia mundial al tratar un paciente ortopédico es realizar las correcciones quirúrgicas lo más pronto posible para evitar el avance del grado de las enfermedades o que se generen complicaciones secundarias (Martí, 2013; Hans et al, 2016), en el país, la mayoría de los pacientes cachorros que presentan anormalidades en el andar son subestimados y aun cuando se diagnostica médicamente alguna lesión ortopédica se prefiere postergar el tratamiento para etapas posteriores de la vida del paciente. Esto debido a que en esta etapa suelen ser cuadros no dolorosos que al parecer solo produce un caminar peculiar en los pacientes y al temor de los riesgos anestésicos en los animales jóvenes. El retraso de las medidas correctivas permite que las lesiones evolucionen y se acompañen de complicaciones secundarias que complican el cuadro del paciente y limitan seriamente la posibilidad de recuperación (DeCamp y Johnson, 2006; Martí, 2013; Cashmore et al., 2014; Kalff et al., 2014; Wangdee et al., 2014; Hans et al., 2016). 
En el caso clínico presentado se evaluó una paciente muy joven con una seria incapacidad para deambular y con una evidente luxación patelar de grado 4, pero con imágenes radiográficas aparentemente normales para un paciente de su edad. Esto podría llevar a confusión, pero ratifica el hecho de que el diagnóstico de esta enfermedad se basa en la evaluación clínica (DeCamp y Johnson, 2006), ya que cuando el paciente apoyaba peso durante su andar, las estructuras que se encontraban no alineadas tomaban posiciones anormales, pero cuando eran traccionadas en posición ventrodorsal parecían normales.

El tratamiento quirúrgico temprano se presenta como una alternativa viable frente a la luxación patelar, ya que no hay una asociación significativa entre las complicaciones posquirúrgicas del tratamiento y la edad al momento de la cirugía (Arthurs y LangleyHobbs, 2006). En opinión de los autores, el hecho de encontrar las estructuras óseas normales en la evaluación radiográfica plantea mucho mejor pronóstico para el paciente a pesar de su joven edad. Esto se demuestra con la evolución del caso clínico donde en el día 15 posquirúrgico presentaba un apoyo de los miembros posteriores casi normal.

\section{Literatura Citada}

1. Alam MR, Lee JI, Kang HS, Kim IS, Park SY, Lee KC, Kim NS. 2007. Frequency and distribution of patellar luxation in dogs: 134 cases (2000 to 2005). Vet Comp Orthop Traumatol 20: 59-64.

2. Arthurs GI, Langley-Hobbs SJ. 2006. Complications associated with corrective surgery for patellar luxation in 109 dogs. Vet Surg 35: 559-566. doi: 10.1111/j.1532950X.2006.00189.x

3. Cashmore R, Havlicek M, Perkins N, James D, Fearnside S, Marchevsky A, Black AP. 2014. Major complications and risk factors associated with surgical correction of congenital medial patellar luxation in 124 dogs. Vet Comp Orthop Traumatol 27: 263-70. doi: 10.3415/ VCOT-13-08-0100

4. DeCamp C, Johnson S. 2006. The stifle joint. In: Brinker, Piermatei and Flo's Handbook of small animal orthopaedics and fracture repair. $4^{\text {th }}$ ed. Philadelphia, USA: Saunders. p 562-582.

5. Hayes AG, Boudrieau RJ, Hungerford LL. 1994. Frequency and distribution of medial and lateral patellar luxation in dogs: 124 cases (1982-1992). J Am Vet Med Assoc 205: 716-720.

6. Hans E, Kerwin S, Elliott AC, Butler R, Saunders WB, Hulse DA. 2016. Outcome following surgical correction of grade 4 medial patellar luxation in dogs: 47 stifles (2001-2012). J Am Anim Hosp Assoc 52: 162-169. doi: 10.5326/ JAAHA-MS-6329

7. KalffS, Butterworth S, Miller A, Keeley B, Baines S, McKee W. 2014. Lateral patellar luxation in dogs: a retrospective study of 65 dogs. Vet Comp Orthop Traumatol 27: 130-134. doi: 10.3415/ VCOT-13-05-0064

8. Martí S. 2013. Enfermedades del desarrollo musculoesquelético. En: Medicina pediátrica en pequeños animales. España: Ed. Servet. p. 193-225.

9. Piermattei DL, Flo GL, DeCamp CE. 2006. The stifle joint. In: Brinker, Piermatei and Flo's Handbook of small animal orthopaedics and fracture repair. $4^{\text {th }}$ ed. Philadelphia, USA: Saunders. $p$ 562-581.

10. Wangdee $C$, Hazewinkel $H$, Temwichitr J, Theyse L. 2014. Extended proximal trochleoplasty for the correction of bidirectional patellar luxation in seven Pomeranian dogs. J Small Anim Pract 56: 130-133. doi: 10.1111/jsap. 12248 